\title{
Novel reference-free methods for the determination of the instrumental response of Laue-type bent crystal spectrometers
}

\begin{abstract}
Monika Szlachetko, ${ }^{a}$ Joanna Hoszowska, ${ }^{a}$ Jakub Szlachetko ${ }^{b}$ and Jean-Claude Dousse (10) *a

We report on novel reference-free methods to determine the instrumental resolution of transmission-type bent crystal spectrometers. The novel methods are based on the measurements of a selected X-ray line in several orders of diffraction. It is shown that the angular broadening of the spectrometer and the natural linewidth of the selected transition can be obtained directly from the novel methods. No reference X-ray line or $\gamma$-ray line is needed. The precision of the results is about 10 times better than the one obtained with the standard method consisting of measuring a reference $X$-ray line and keeping fixed in the fit the natural width of this line at the value taken from available databases. The novel methods are illustrated for a DuMond-type bent crystal spectrometer with the $K \alpha_{1} X$-ray line of Gd measured in the first five orders of diffraction.
\end{abstract}

\section{Introduction}

In high energy resolution X-ray spectroscopy using crystal spectrometers, an accurate knowledge of the instrumental broadening of the spectrometer is crucial for correct and reliable fits of the measured spectra. In particular, in projects aiming to determine the natural linewidths of X-ray transitions, the knowledge of the instrumental resolution as a function of the photon energy is mandatory to deconvolve properly the Voigtian profiles of the measured X-ray lines and to obtain accurate results for the Lorenztian widths of the latter. ${ }^{1-5}$ For both Laue- and Bragg-type bent crystal spectrometers, ${ }^{6}$ the instrumental response is usually well reproduced by a Gaussian function, ${ }^{1,7}$ whereas the shape of an X-ray line is described by a Lorentzian function whose full width at half maximum (FWHM) reflects the natural lifetime broadening of the corresponding radiative transition. X-ray spectra are therefore fitted using Voigt functions ${ }^{8}$ since the latter result from the convolution of the Gaussian response of the spectrometer with the Lorentzian shape of X-ray lines. In the fit of an angular X-ray spectrum, the angular instrumental broadening of the spectrometer is in general kept fixed at its known value for all X-ray lines, whereas the Bragg angles corresponding to the centroid positions of the lines, the intensities and Lorentzian widths of

${ }^{a}$ Physics Department, University of Fribourg, Ch. du Musée 3, CH-1700 Fribourg, Switzerland. E-mail: jeanclaude.dousse@unifr.ch; Fax: +41 26 3009747; Tel: +41 26 3009073

${ }^{b}$ Institue of Nuclear Physics, Polish Academy of Science, Radzikowskiego 152, PL-31342 Kraków, Poland the latter as well as the slope and intercept of the linear background are used as free fitting parameters.

In this paper we report on two novel reference-free methods to determine the angular resolution (and consequently the energy resolution) of Laue-type (also referred to as transmission-type) bent crystal spectrometers. The Dumond ${ }^{9,10}$ and Cauchois ${ }^{11}$ geometries are the most commonly used setups for transmission-type spectrometers. They both require a cylindrically bent crystal with a radius of curvature $R$ which is equal to the diameter $2 R$ of the focal Rowland circle. In the DuMond geometry, the point-like source is placed on the Rowland circle, while the extended detector is located outside of the Rowland circle on the other side of the crystal. The Cauchois geometry is the reverse of the DuMond geometry. In the Cauchois setup, the small size or position sensitive detector is placed on the Rowland circle while the extended source is placed outside of the latter. In both setups, a Soller slit collimator is usually inserted between the crystal and the detector (DuMond case) or source (Cauchois case) in order to shield the detector from the direct radiation from the source. More details about the two setups can be found in ref. 6 and 12 .

The two reference-free methods presented in this paper are based on the measurements of a selected characteristic X-ray line measured in several diffraction orders. The angular range covered by Laue-type crystal spectrometers extends usually from a few degrees or less up to about $20^{\circ}$ or more so that several diffraction orders can be easily measured with the same crystal. For instance, for the Dumond spectrometer of Fribourg, ${ }^{13}$ whose usable angular range extends from $0.17^{\circ}$ up to $20^{\circ}$, the use of $\mathrm{SiO}_{2}(110)$ crystal permits to measure the $\mathrm{K} \alpha_{1} \mathrm{X}$-ray line of gold $(E=68.804 \mathrm{keV})$ at diffraction orders varying from $n=1$ 
(Bragg angle $\left.\vartheta=2.102^{\circ}\right)$ up to $n=9\left(\vartheta=19.274^{\circ}\right)$. The chosen $\mathrm{X}$-ray line must be intense and perfectly symmetric. In addition, to get precise and reliable results, at least three different orders of diffraction should be measurable (see Section 4.2). For these reasons, $\mathrm{K} \alpha \mathrm{X}$-ray lines of elements with $Z \geq \approx 45$ should be preferably chosen (the minimum atomic number $Z$ depends on the maximum Bragg angle that can be scanned by the employed spectrometer).

The novel reference-free methods are explained by means of measurements performed with the Laue-type bent crystal spectrometer of Fribourg. ${ }^{13}$ The latter was developed for inhouse photoinduced X-ray emission spectroscopy (XES) measurements. It is based on the so-called modified DuMond slit geometry. In this geometry a narrow rectangular slit placed on the focal circle in front of the sample serves as effective source of radiation (see Fig. 1 in ref. 13). The slit width which influences in opposite ways the resolution and luminosity of the spectrometer can be adjusted. In most applications slit widths between $0.05 \mathrm{~mm}$ and $0.15 \mathrm{~mm}$ are employed. For XES measurements, the spectrometer is equipped with a $10 \mathrm{~cm}$ wide $\times 10 \mathrm{~cm}$ high $\times 0.5 \mathrm{~mm}$ thick $\mathrm{SiO}_{2}(110)$ crystal $\left[2 d_{110}=4.913\right.$ $\AA$ ํ. The effective reflecting area which is limited by the bending device amounts to $12 \mathrm{~cm}^{2}$. The Bragg angle domain covered by the spectrometer extends from $-20^{\circ}$ to $+20^{\circ}$. For each diffraction order, the X-ray lines can be measured on both sides of reflection, i.e., at positive and negative Bragg angles, permitting thus an accurate determination of the origin of the Bragg angles scale. This is a necessary prerequisite for a reliable energy calibration of the spectrometer. A two-stage worm gear tooth wheel system driven by a stepping motor allows to carry out angular steps as small as $0.075 \operatorname{arcsec} \cong 0.36 \mu$ rad but in most XES measurements angular steps of about 3 arcsec $\cong 14.5 \mu \mathrm{rad}$ are used. The Bragg angles are measured by means of a Doppler-

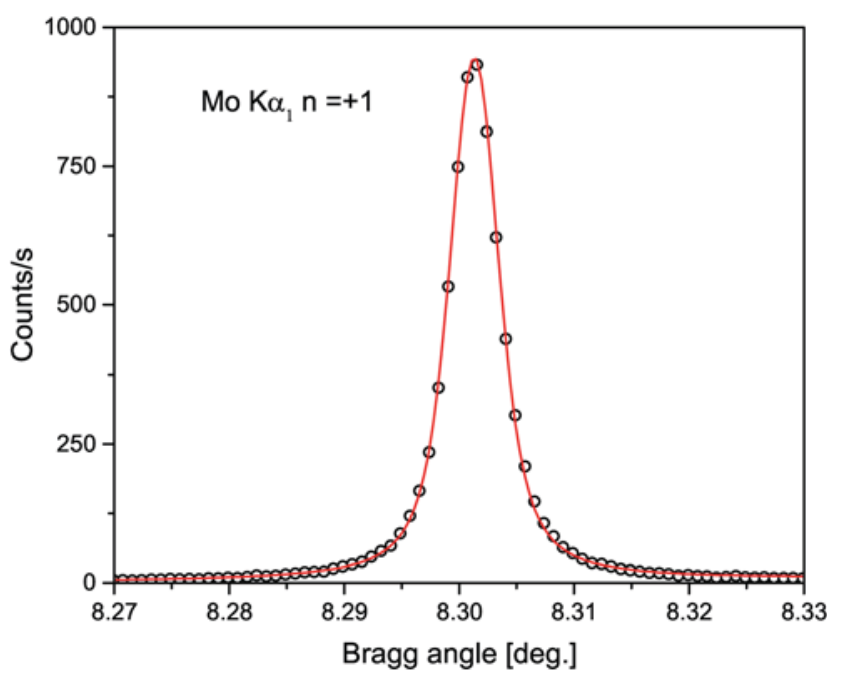

Fig. 1 (Color online): Angular spectrum of the Mo $\mathrm{K} \alpha_{1} \mathrm{X}$-ray emission line measured in first order of diffraction with a slit width of $0.10 \mathrm{~mm}$. The open circles represent the measured points (step by step measurement) and the solid line stands for the total fit, i.e., the sum of the fitted Voigtian and linear background. shift based laser interferometer ${ }^{14}$ with an absolute accuracy of 5 $\times 10^{-3}$ arcsec $\cong 0.024 \mu \mathrm{rad}$.

The paper is organized as follows. At first, readers not familiar with crystal spectroscopy are reminded of some basic principles concerning the angular and energy resolution of crystal spectrometers (Section 2). Then, standard methods which are routinely employed for the determination of the instrumental response of crystal spectrometers are presented (Section 3). In Section 4, the two reference-free methods are described in details and explained. The results obtained with the standard and reference-free methods are compared and discussed in Section 5 where the issue of applying the referencefree methods to Bragg-type crystal spectrometers is also addressed. Finally, concluding remarks and a brief outlook are presented in Section 6.

\section{Angular and energy instrumental broadenings}

The FWHM angular resolution $\Gamma_{\mathrm{inst}, \vartheta}$ of a DuMond-type crystal spectrometer depends in decreasing order of importance on the precision of the crystal curvature, source or slit width and Darwin width of the crystal. In the Cauchois case, the source/slit width should be replaced by the detector size or detector spatial resolution if a position-sensitive detector is used. The instrumental response which results from the convolution of the angular distributions corresponding to the three sources of broadening can be well reproduced by a Gaussian function (see, e.g., ref. 1). In first approximation the angular resolution can be considered as nearly constant over the entire angular domain covered by the spectrometer. Actually, the precision of the crystal curvature does not depend on the Bragg angle, and only tiny changes with the Bragg angle are observed in the angular broadenings related to the source/slit width and Darwin width.

The source/slit width broadening reads:

$$
\Gamma_{\mathrm{s}}=2 \arctan \frac{w_{\mathrm{s}}}{2 f},
$$

where $w_{\mathrm{s}}$ stands for the source or slit width and $f$ is the focal distance, i.e., the distance between the crystal and the source/ slit. For the Bragg angle $\vartheta$ the latter is given by:

$$
f=R \cos (\vartheta)
$$

where $R$ represents the radius of curvature of the crystal. For the DuMond spectrometer of Fribourg, $R \cong 316 \mathrm{~cm}$ (ref. 13) so that for $w_{\mathrm{s}}=0.1 \mathrm{~mm}$, the slit-induced broadening corresponding to the $\mathrm{K} \alpha_{1} \mathrm{X}$-ray line of $\mathrm{Gd}(E=42.996 \mathrm{keV})$ amounts to $31.7 \mu \mathrm{rad}$ in first order of diffraction $\left(\vartheta \cong 3.4^{\circ}\right)$ and $33.1 \mu \mathrm{rad}$ in fifth order of diffraction $\left(\vartheta \cong 17.1^{\circ}\right)$. The Darwin width $\Gamma_{\mathrm{D}}$ depends on the energy of the diffracted X-rays but for a given energy it varies only slightly with the diffraction order, i.e., the Bragg angle. The Darwin width can be calculated with the computer code XOP. ${ }^{15}$ For the above example $\left(\mathrm{K} \alpha_{1}\right.$ X-ray line of $\mathrm{Gd}$ measured in first and fifth orders of diffraction) the Darwin widths calculated with the XOP code amount to $2.4 \mu \mathrm{rad}$ and 2.2 $\mu \mathrm{rad}$, respectively. 
The FWHM energy resolution $\Gamma_{\text {inst }, E}$ of the spectrometer can be deduced from the FWHM angular resolution $\Gamma_{\mathrm{inst}, \vartheta}$ via the Bragg law. One finds:

$$
\Gamma_{\text {inst }, E}=\cot (\vartheta) \Gamma_{\text {inst }, \vartheta} E,
$$

where $E$ is the energy of the observed X-ray line and $\vartheta$ the corresponding Bragg angle. Using eqn (3) and the value of about $56.7 \mu \mathrm{rad}$ for $\Gamma_{\mathrm{inst}, \vartheta}$ (see next Section), an energy resolution $\Gamma_{\text {inst }, E}=6.8 \mathrm{eV}$ is found for the Mo K $\alpha_{1}$ X-ray line $(E=17.479$ $\mathrm{keV}$ ) in first order of diffraction, whereas for the $\mathrm{Gd} \mathrm{K \alpha _{1 }}$ X-ray line $(E=42.996 \mathrm{keV})$ in first order of diffraction, the energy resolution amounts to $41.5 \mathrm{eV}$. This illustrates the fact that for crystal spectrometers the energy resolution becomes poorer with increasing photon energy. Actually, from eqn (3), one can show that the energy resolution deteriorates approximately with the squared energy of the measured photons:

$$
\Gamma_{\text {inst }, E}=\frac{\cos (\vartheta)}{\sin (\vartheta)} \Gamma_{\text {inst }, \vartheta} E=\frac{\cos (\vartheta)}{\frac{h c}{2 d_{\mathrm{hkl}}} \frac{n}{E}} \Gamma_{\mathrm{inst}, \vartheta} E \cong \frac{2 d_{\mathrm{hkl}}}{n h c} \Gamma_{\mathrm{inst}, \vartheta} E^{2},
$$

where $h$ stands for the Planck constant, $c$ for the velocity of light in vacuum, $d_{\mathrm{hkl}}$ for the spacing constant of the diffraction planes (hkl) and $n$ for the diffraction order. For Bragg angles $\vartheta$ $\leq \approx 18^{\circ}$, the error related to the approximation $\cos (\vartheta) \approx 1$ done in eqn (4) is smaller than $5 \%$ so that, for a given diffraction order and instrumental angular resolution, the instrumental energy resolution can be considered to be indeed nearly proportional to $E^{2}$.

\section{Standard methods}

\subsection{X-ray line as reference}

The standard method to determine the angular instrumental broadening of Laue-type or Bragg-type bent crystal spectrometers consists to measure a strong X-ray line and to keep fixed in the fit of the measured angular spectrum the Lorentzian width at its known value. ${ }^{13,16}$ For bent crystal spectrometers installed at synchrotron radiation facilities, the elastic peak of the beam can also be used as reference line provided that the energy resolution of the beam is accurately known. ${ }^{17-19}$ The natural width of the reference transition can be deduced from the data base of Salem and $\mathrm{Lee}^{20}$ or preferably from the more recent one of Campbell and Papp. ${ }^{21}$ In these data bases, the widths are reported in $\mathrm{eV}$. The latter, however, can be converted into angular widths $\Gamma_{\text {nat }, \vartheta}$ using the following simple relation:

$$
\Gamma_{\mathrm{nat}, \vartheta}=\tan (\vartheta) \frac{\Gamma_{\mathrm{nat}, E}}{E},
$$

where $\vartheta$ is the Bragg angle corresponding to the reference transition of energy $E$ and $\Gamma_{\text {nat }, E}$ the natural linewidth of this transition. In the above relation, the same units should be used for $E$ and $\Gamma_{\text {nat }, E}$ so that the value obtained for $\Gamma_{\text {nat }, \vartheta}$ corresponds to $\operatorname{rad}$ (or $\mu \mathrm{rad}$ ).

The standard method is discussed in more details hereafter for the case of the $\mathrm{K} \alpha_{1}$ X-ray line of Mo $(E=17.479 \mathrm{keV})$ measured with the DuMond spectrometer of Fribourg. The target fluorescence was produced by irradiating a $53 \mathrm{mg} \mathrm{cm} \mathrm{cm}^{-2}$ thick Mo metallic foil with the Bremsstrahlung of a side-window $\mathrm{Au}$ anode X-ray tube operated at $80 \mathrm{kV} \times 30 \mathrm{~mA}$. The measurement was performed in first order of diffraction with a slit width of $0.1 \mathrm{~mm}$ and an acquisition time of $20 \mathrm{~s}$ per angular step.

The spectrum was analysed by means of the least-squares fitting program PeakFit (SYSTAT Software Inc., Richmond, USA), employing a Voigt function to fit the $\mathrm{K} \alpha_{1}$ line (see Fig. 1). The centroid angular position of the line, its intensity, the standard deviation $\sigma_{\text {inst }, \vartheta}=\Gamma_{\text {inst }, \vartheta} /(2 \sqrt{2 \ln 2})$ of the Gaussian instrumental response and the two parameters of the linear background were let free in the fit, whereas the Lorentzian width was kept fixed at the value derived from the atomic level widths of the $K$ and $L_{3}$ levels quoted in ref. 21, using eqn (5) for the transformation of the latter in angular widths. From the fit a value of $0.001379(13)^{\circ}=24.07(23) \mu \mathrm{rad}$ was obtained for $\sigma_{\text {inst }, \vartheta}$. The corresponding FWHM $\Gamma_{\text {inst }, \vartheta}$ amounts thus to about $56.7 \mu \mathrm{rad}$. As for this transition $\Gamma_{\mathrm{s}}=32.0 \mu \mathrm{rad}$ and $\Gamma_{\mathrm{D}}=5.0$ $\mu \mathrm{rad}$, one deduces from the deconvolution that the contribution of the crystal curvature to the total instrumental broadening is approximately $51.7 \mu \mathrm{rad}$.

To check the possible influence of the $\mathrm{K} \alpha_{2}$ line $(E=17.374$ $\mathrm{keV}$ ) on the fit of the $\mathrm{K} \alpha_{1}$, a separate fit of the whole $K \alpha_{1,2}$ spectrum was performed. In this fit the natural widths of the two lines were kept fixed at the values derived from the Tables of Campbell and Papp $^{21}$ and the instrumental broadening $\sigma_{\text {inst }, \vartheta}$ was let free. The same instrumental broadening could be used for both lines, thanks to the parameter sharing option provided by the PeakFit program. The difference between the values of $\sigma_{\text {inst }, \vartheta}$ provided by the $K \alpha_{1,2}$ and $K \alpha_{1}$ fits was found to be negligibly small (about 7 times smaller than the combined error of the two fits).

One might think that $\sigma_{\text {inst }, \vartheta}$ and $\Gamma_{\text {nat }, \vartheta}$ could be used both as free fitting parameters. The fits show, however, that this way of proceeding may lead to inaccurate results and that one of the two widths has to be anchored to a fixed value to get a reliable result for the second width. For instance, letting both width parameters free in the fit of the Mo $\mathrm{K} \alpha_{1}$ spectrum, values of 20.83(20) $\mu \mathrm{rad}$ and 59.44(43) $\mu \mathrm{rad}$ are obtained for $\sigma_{\text {inst }, \vartheta}$ and $\Gamma_{\text {nat, }, \vartheta}$, respectively. One sees that the difference between the values of $\sigma_{\text {inst }, \vartheta}$ provided by the two fits $(3.24 \mu \mathrm{rad})$ is more than 10 times bigger than their combined error $( \pm 0.3 \mu \mathrm{rad})$, whereas the Lorentzian width in eV corresponding to 59.44(43) $\mu \mathrm{rad}$ is $7.12(5) \mathrm{eV}$, a result which is clearly not consistent either with the value of $6.21(31) \mathrm{eV}$ derived from the atomic level widths recommended by Campbell and Papp. ${ }^{21}$

The above mentioned uncertainty on the angular instrumental resolution $\left(\Delta \sigma_{\text {inst }, \vartheta}= \pm 0.23 \mu \mathrm{rad}\right)$ obtained with the standard method using the known natural width of the $\mathrm{K} \alpha_{1}$ transition of Mo corresponds to the error given by the fitting procedure for the free parameter $\sigma_{\text {inst }, \vartheta}$. The uncertainty on the natural width of the transition is not included. If the latter is considered $\left(\Delta \Gamma_{\text {nat }, E}= \pm 0.31 \mathrm{eV}\right.$ (ref. 21)), the error becomes significantly bigger, namely $\Delta \sigma_{\text {inst }, \vartheta}= \pm 0.89 \mu \mathrm{rad}$. The precision of the method is thus limited by the uncertainty of the 
natural width of the transition used as reference and the accuracy of the so-determined angular instrumental broadening by the reliability of the transition width used as benchmark.

The value of 24.07(89) $\mu \mathrm{rad}$ obtained for the angular instrumental broadening from the measurement of the $\mathrm{K} \alpha_{1}$ transition of Mo was cross-checked with the $K \alpha_{1}$ transition of Gd $(E=42.996 \mathrm{keV})$ measured in first order of diffraction. In the fit the natural width of the line was kept fixed at the value of $35.72 \mu \mathrm{rad}$ derived, using eqn (5), from the linewidth of $26.12 \mathrm{eV}$ taken from ref. 21. The corresponding spectrum is depicted on the left side of the top panel of Fig. 2. From the fit a value $\sigma_{\text {inst }, \vartheta}$ $=23.05 \pm 0.11 \mu \mathrm{rad}$ was obtained. If one considers the uncertainty $\Delta \Gamma_{\text {nat }, E}= \pm 1.77 \mathrm{eV}$ quoted in ref. 21 for the $\mathrm{K} \alpha_{1}$ transition of $\mathrm{Gd}$, the error $\Delta \sigma_{\text {inst }, \vartheta}$ increases to $0.73 \mu \mathrm{rad}$ and the result is $\sigma_{\text {inst }, \vartheta}=(23.05 \pm 0.73) \mathrm{eV}$, a value which is well in line with the result obtained from the Mo $\mathrm{K} \alpha_{1}$ measurement.

\section{2 $\gamma$-ray line as reference}

To circumvent the problems related to the above-mentioned limited precision and accuracy of the linewidth of the reference X-ray transition, $\gamma$-rays can be used as references instead of $\mathrm{X}$-rays. The natural linewidths of $\gamma$-rays are indeed nearly-zero due to the much longer lifetime of the excited nuclear states as compared to the atomic ones. However, due to the low
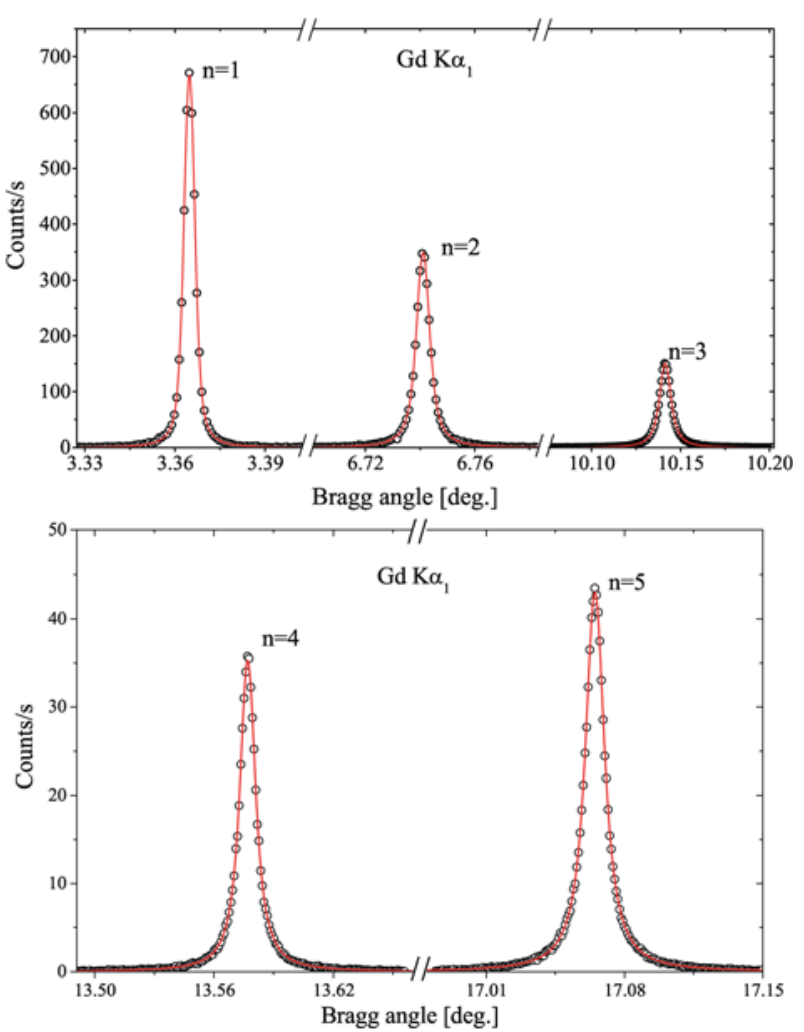

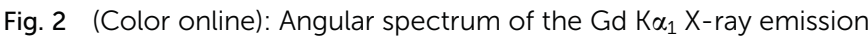
line measured in the first five orders of diffraction with a slit width of $0.10 \mathrm{~mm}$. For each diffraction order, the open circles represent the measured points and the solid line stands for the total fit, i.e., the sum of the fitted Voigtian and linear background. efficiency of crystal spectrometers, very intense radioactive $\gamma$ sources with activities of $\approx 10^{10} \mathrm{~Bq}$ are needed. Such sources can be produced by irradiating enriched isotopes with thermal neutrons provided that the chosen isotopes have large neutroncapture cross sections and the formed unstable isotopes long enough half-lives (several weeks).

For instance, this method was employed for the determination of the experimental broadening of the DuMond spectrometer of Fribourg in a project aiming at determining the energies and linewidths of most $L$ X-ray lines of uranium. ${ }^{1}$ In this work the angular instrumental resolution was determined from the measurement of the $63.121 \mathrm{keV} \gamma$-ray line from the ${ }^{169} \mathrm{Yb} \rightarrow{ }^{169} \mathrm{Tm}$ electron-capture decay. The ${ }^{169} \mathrm{Yb}$ radioactive source (half-life of 32 days) was produced by irradiating with thermal neutrons a powder sample of natural ytterbium enriched to $10 \%$ with ${ }^{168} \mathrm{Yb}$. The measurement was performed in third order of diffraction with a $1 \mathrm{~mm}$ thick $\mathrm{SiO}_{2}(110)$ crystal curved to a radius of $313 \mathrm{~cm}$ using a slit width of $0.15 \mathrm{~mm}$. From the fit of the $\gamma$-ray line with a pure Gaussian function, a FWHM angular resolution of $11.5 \operatorname{arcsec}=55.8 \mu \mathrm{rad}$, corresponding to $\sigma_{\text {inst }, \vartheta}=23.7 \mu \mathrm{rad}$, was obtained.

Above result cannot be directly compared to the ones obtained with the $\mathrm{K} \alpha_{1} \mathrm{X}$-ray lines of Mo and Gd (see Section 3.1) because the X-ray and $\gamma$-ray measurements were performed with two different $\mathrm{SiO}_{2}$ crystals and different slit widths. Nevertheless, considering that in the $\gamma$-ray measurement the slit-induced broadening and the Darwin width amount to $48.3 \mu \mathrm{rad}$ and 1.1 $\mu \mathrm{rad}$, respectively, one finds that the contribution of the crystal curvature was $41.5 \mu \mathrm{rad}$, i.e., about $10 \mu \mathrm{rad}$ smaller than for the $0.5 \mathrm{~mm}$ thick crystal. This is, however, not surprising since it is well known that the accuracy of the bending increases in general with the thickness of the crystal lamina.

The transportation of strong radioactive sources from the nuclear reactor to the laboratory is onerous and complicated because of the stringent safety constraints related to the transportation of radioactive materials. In addition, the lifetimes of such sources are limited to several weeks and enriched isotopes are in most cases rather expensive. For all these reasons, the method based on reference $\gamma$-rays does not represent the ideal solution either.

\section{Reference-free methods}

\subsection{Simultaneous fitting of a selected X-ray line measured in several diffraction orders}

In order to get rid of the uncertainty and possible inaccuracy of the linewidth of the reference transition used in the standard method, a novel approach was developed in which a selected Xray transition is measured in several orders of diffraction and the partial angular spectra corresponding to the different diffraction orders are fitted simultaneously by means of the PeakFit program. For each diffraction order $n$, the angular instrumental broadening is assumed to be the same (see Section 2) and the natural linewidth $\Gamma_{\text {nat, } \vartheta}(n=i)$ is expressed as a function of $\Gamma_{\text {nat }, \vartheta}(n=1)$, using the following expression derived from eqn (5): 


$$
\Gamma_{\text {nat }, \vartheta}(n=i)=\frac{\tan [\vartheta(n=i)]}{\tan [\vartheta(n=1)]} \Gamma_{\text {nat }, \vartheta}(n=1) .
$$

At first the linear background of each line is determined and subtracted from the measured points. Taking then benefit of the possibility provided by the PeakFit program to fit the data with an user defined function (UDF), the lines corresponding to the $m$ different diffraction orders are fitted by means of the following UDF:

$$
F(\vartheta)=\sum_{i=1}^{m} \frac{a_{0, i}}{\sqrt{2} \pi \sigma q_{i} \Gamma_{\mathrm{nat}}} \int_{0}^{\infty} \mathrm{e}^{-\frac{1}{2}\left(\frac{\vartheta-\vartheta_{i}}{\sigma}\right)^{2}} \frac{1}{1+\left(\frac{\vartheta-\vartheta_{i}-t}{q_{i} \Gamma_{\text {nat }}}\right)^{2}} \mathrm{~d} t
$$

where $a_{0, i}$ stands for the area under the peak corresponding to the $i$-th diffraction order, $\sigma=\sigma_{\text {inst }, \vartheta}, \Gamma_{\text {nat }}=\Gamma_{\text {nat }, \vartheta}(n=1)$ and $q_{i}=$ $\tan \left(\vartheta_{i}\right) / \tan \left(\vartheta_{1}\right)$. In the fit, the parameters $a_{0, i}$, the line centroids $\vartheta_{i}$, as well as the two parameters of interest $\sigma$ and $\Gamma_{\text {nat }}$ are let free with the constraint that the last two parameters are the same for all diffraction orders, using the share option provided by the PeakFit code. In general, for the first and second diffraction orders the angular full width at half maximum of the measured line is dominated by the Gaussian width, whereas for higher diffraction orders the Lorentian width prevails due to the coefficient $q_{i}$ so that, in constrast with the standard method, both width parameters can be let free in the fit of the angular multi-line spectrum.

The novel method was applied to the $\mathrm{K} \alpha_{1} \mathrm{X}$-ray line of $\mathrm{Gd}$ measured in the first five orders of diffraction. The measurements were performed with a $36 \mathrm{mg} \mathrm{cm}^{-2}$ thick metallic foil irradiated with the bremsstrahlung produced by a Au anode Xray tube which was operated at $80 \mathrm{kV}$ and $30 \mathrm{~mA}$. The same slit width of $0.10 \mathrm{~mm}$ was used. Due to the decreasing reflectivity of the crystal with the diffraction order, collecting times of $10 \mathrm{~s}$, $20 \mathrm{~s}, 40 \mathrm{~s}, 50 \mathrm{~s}$ and $70 \mathrm{~s}$ per step were used for the orders 1, 2, 3, 4 and 5, respectively. The partial angular spectra were scanned using 100 steps for $n=1$ and 2, 150 steps for $n=3$ and 200 steps for $n=4$ and 5 with a step length of 3 arcsec. for each diffraction order.

The fit of the angular spectrum corresponding to the five orders of diffraction is depicted in Fig. 2. The fit was performed with the function $F(\vartheta)$ defined in eqn (7). As shown by Fig. 2, the intensity diminishes with the diffraction order as expected except for the 5th order which is more intense than the 4 th order. This apparent anomaly is due to the fact that the diffracted intensity increases with the structure factor $F_{\mathrm{hkl}}$ and that the latter, for the employed $\mathrm{SiO}_{2}$ (110) crystal and the energy of $42.996 \mathrm{keV}$, is bigger for the 5 th order $\left(F_{550}=15.0\right)$ than for the 4 th one $\left(F_{440}=8.3\right)$. From the simultaneous fit of the five lines the following values were obtained: $\sigma=0.001318(4)^{\circ}$ and $\Gamma_{\text {nat }}=$ $0.002051(8)^{\circ}$. Rewriting these values in the proper units and using eqn (3) to transform $\Gamma_{\text {nat }}$ in $\mathrm{eV}$, one finds: $\sigma_{\text {inst }, \vartheta}=23.00$ (7) $\mu \mathrm{rad}$ and $\Gamma_{\text {nat }, E}=26.18(10) \mathrm{eV}$. The numbers indicated in brackets correspond to the errors provided by the fit.
4.2 Instrumental angular broadening from the full widths at half maximum of a selected X-ray line measured in several diffraction orders

In this method, a chosen fluorescence X-ray line is also measured in different orders of diffraction. The standard deviation of the Gaussian representing the angular instrumental broadening and the natural angular width of the selected transition are derived from the full widths at half maximum of the lines corresponding to the different diffraction orders. It is again assumed that the angular instrumental broadening does not depend on the Bragg angle and is thus the same for all diffraction orders, while the angular natural width of the selected transition measured in the $i$ th order of diffraction is written, according to eqn (6), as $\Gamma_{\text {nat }, \vartheta}(n=i)=q_{i} \Gamma_{\text {nat }, \vartheta}(n=1)$. Now, since for each diffraction order the known full width at half maximum of the measured line is equal to the width resulting from the convolution of the Gaussian instrumental response with the Lorentzian shape of the transition, a system of equations with two unknowns, namely $\sigma_{\text {inst }, \vartheta}$ and $\Gamma_{\text {nat }, \vartheta}$, can be obtained from the set of measurements. To solve the system of equations, the transition has to be measured at least in two different diffraction orders. However, the method is more accurate if the measurements are carried out in more than two orders of diffraction and the overdetermined system of equations is solved by means of a non-linear least-squares fitting method.

Mathematically, this method consists in minimizing the function $S\left(\sigma, \Gamma_{\text {nat }}\right)$ (we use again the simplified notations $\sigma=$ $\sigma_{\text {inst }, \vartheta}$ and $\left.\Gamma_{\text {nat }}=\Gamma_{\text {nat }, \vartheta}\right)$ given by:

$$
S\left(\sigma, \Gamma_{\mathrm{nat}}\right)=\sum_{i=1}^{m}\left[\frac{\Gamma_{\mathrm{obs}, i}-V_{i}\left(\sigma, \Gamma_{\mathrm{nat}}\right)}{\Delta \Gamma_{\mathrm{obs}, i}}\right]^{2},
$$

where $m$ represents the number of X-ray lines measured in different diffraction orders, $\Gamma_{\mathrm{obs}, i}$ the full width at half maximum of the line measured in the $i$-th order of diffraction, $\Delta \Gamma_{\mathrm{obs}, i}$ the uncertainty of $\Gamma_{\mathrm{obs}, i}$ and $V_{i}\left(\sigma, \Gamma_{\text {nat }}\right)$ the width of the Voigtian resulting from the folding of the Gaussian having a standard deviation $\sigma$ with the Lorentzian having a width $q_{i} \Gamma_{\text {nat. }}$

The solutions of the overdetermined system can be found by solving the following system of equations:

$$
\begin{gathered}
\frac{\partial S\left(b_{1}, b_{2}\right)}{\partial b_{1}}=0 \\
\frac{\partial S\left(b_{1}, b_{2}\right)}{\partial b_{2}}=0,
\end{gathered}
$$

where $b_{1}=\sigma$ and $b_{2}=\Gamma_{\text {nat }}$.

The uncertainties of the solutions are given by the so-called covariance or error matrix $U$ defined by:

$$
\left(U^{-1}\right)_{i, j}=\frac{1}{2} \frac{\partial^{2} S\left(b_{i}, b_{j}\right)}{\partial b_{i} \partial b_{j}} .
$$

The second derivatives which form the inverse of the error matrix are evaluated at the minimum. The diagonal elements 
$U_{i i}$ correspond to the variances of the variables $b_{i}$, while the offdiagonal elements $U_{i j}$ represent the covariances between $b_{i}$ and $b_{j}$. Thus,

$$
U=\left(\begin{array}{cc}
\Delta \sigma^{2} & \operatorname{cov}\left(\sigma, \Gamma_{\text {nat }}\right) \\
\operatorname{cov}\left(\Gamma_{\text {nat }}, \sigma\right) & \Delta \Gamma_{\text {nat }}^{2}
\end{array}\right)
$$

Note that $\operatorname{cov}\left(\sigma, \Gamma_{\text {nat }}\right)=\operatorname{cov}\left(\Gamma_{\text {nat }}, \sigma\right)$ since the error matrix $U$ is symmetric.

The Voigtian widths $V_{i}$ were determined using the following approximation: ${ }^{22}$

$$
V_{i}=0.5346 \Gamma_{i}+\sqrt{0.2166 \Gamma_{i}^{2}+5.545 \sigma^{2}},
$$

where $\Gamma_{i}$ stands for the angular Lorentzian width of the line measured at the diffraction order $i$ and $\sigma$ for the angular standard deviation of the Gaussian instrumental response. The accuracy of the approximation was checked by comparing the widths $V_{i}$ obtained by convolution (exact values) and by means of the relation (12) (approximate values), using the results $\sigma=$ $23.08 \mu \mathrm{rad}$ and $\Gamma_{\text {nat }}=35.30 \mu \mathrm{rad}$ obtained with the second reference-free method (see below) and the coefficients $q_{i}$ listed in Table 1. As shown in Table 1 all relative deviations between $V_{i}^{\text {conv }}$ and $V_{i}^{\text {approx }}$ are smaller than $200 \mathrm{ppm}$, in agreement with the accuracy of $0.02 \%$ claimed by the authors of ref. 22 .

For illustration, the second reference-free method was applied to the same set of measurements as the first one, namely to the $\mathrm{Gd} \mathrm{K} \alpha_{1} \mathrm{X}$-ray line measured in five diffraction orders. As the full width at half maximum of a fitted line is also provided by the Peakfit program, the values $\Gamma_{\mathrm{obs}, i}$ and the uncertainties of the latter were obtained by fitting separately the line associated to each diffraction order. The so-obtained widths $\Gamma_{\mathrm{obs}, i}$ are presented in Table 1. Inserting the approximation (12) in eqn (8) the system of eqn (9) can be solved analytically in a straightforward way. The solutions are $\sigma=$ $23.08 \mu \mathrm{rad}$ and $\Gamma=35.30 \mu \mathrm{rad}$. The variances of the two solutions are calculated from the error matrix (10) at the minimum of $S$, i.e., by using the known values of the solutions in the second derivatives. The following error matrix is obtained:

$$
U=\left(\begin{array}{cc}
4.717 \times 10^{-3} & -3.069 \times 10^{-3} \\
-3.069 \times 10^{-3} & 3.424 \times 10^{-3}
\end{array}\right)
$$

Using eqn (3) to transform $\Gamma_{\text {nat }}$ and $\Delta \Gamma_{\text {nat }}$ in $\mathrm{eV}$, the following results are thus obtained by means of the second reference-free method: $\sigma_{\text {inst }, \vartheta}=(23.08 \pm 0.07) \mu \mathrm{rad}$ and $\Gamma_{\mathrm{nat}, E}=(25.82 \pm$ $0.04) \mathrm{eV}$.

\section{Results and discussion}

The results obtained with the different methods are summarized in Table 2. The value of $\sigma_{\text {inst, } \vartheta}$ deduced from the measurement of the $63.121 \mathrm{keV} \gamma$-ray line from the ${ }^{169} \mathrm{Yb}$ decay (see Section 3.2), however, is not included because this line was measured with another crystal and a different slit width.

A rapid inspection of Table 2 shows first that the standard deviations $\sigma_{\text {inst }, \theta}$ of the Gaussian instrumental broadening of 24.07(89) and 23.05(73) $\mu \mathrm{rad}$ obtained with the standard method using the Mo K $\alpha_{1} n=1$ and Gd $\mathrm{K} \alpha_{1} n=1$ reference Xray lines differ by $1.02 \mu \mathrm{rad}$, whereas the uncertainty on the difference amounts to $1.15 \mu \mathrm{rad}$; the two results are thus consistent.

As it can be also seen in Table 2, the results obtained with the reference-free methods for $\sigma_{\text {inst, } \theta}$ are well in line with those provided by the standard method and about 10 times more precise than the latter. Furthermore, the results provided by the two reference-free methods are consistent regarding $\sigma_{\text {inst }, \theta}$ but not regarding $\Gamma_{\text {nat }, E}$, the deviation of the two values being about 3.3 times bigger than the uncertainty on their difference. It can be noted, however, that although the two reference-free methods do not provide consistent values for the Lorentzian width of the selected X-ray line, both results agree well with the value of $(26.12 \pm 1.77) \mathrm{eV}$ deduced from the atomic level widths reported by Cambell and Papp, ${ }^{21}$ due to the rather large uncertainties reported by these authors for the widths of the $K$ $( \pm 5-10 \%$ for elements with $30 \leq Z \leq 92)$ and $L_{3}( \pm 10 \%$ for elements $50 \leq Z \leq 92$ ) atomic levels.

The discrepancy between the natural Lorentzian widths provided by the two reference-free methods is probably due to the lower intensities of the lines diffracted at higher diffraction orders. Actually, in the first method the least-squares fitting program minimalizes the sum of the squared differences between the measured counts and the calculated ones. As the lines corresponding to $n=4$ and $n=5$ are much less intense, the minimalization process is less sensitive for these orders

Table 2 Standard deviation $\sigma_{\text {inst }, \vartheta}$ of the Gaussian angular instrumental broadening of the DuMond spectrometer equipped with a $0.5 \mathrm{~mm}$ thick $\mathrm{SiO}_{2}$ (110) crystal and a slit with of $0.10 \mathrm{~mm}$ as inferred from the different methods. For the reference-free methods, the natural width of the measured transition could be also determined. The results presented in the 4th row (Ref.-free \#1 cor.) were obtained from normalized intensities (see text)

\begin{tabular}{llrr}
\hline Method & Measurement & $\sigma_{\text {inst }, \vartheta}[\mu \mathrm{rad}]$ & \multicolumn{1}{c}{$\Gamma_{\text {nat }, E}[\mathrm{eV}]$} \\
\hline Standard & $\mathrm{K} \alpha_{1}$ Mo $n=1$ & $24.07 \pm 0.89$ & $6.21 \pm 0.31^{21}$ \\
Standard & $\mathrm{K} \alpha_{1} \mathrm{Gd} n=1$ & $23.05 \pm 0.73$ & $26.12 \pm 1.77^{21}$ \\
Ref.-free \#1 & $\mathrm{K} \alpha_{1} \mathrm{Gd} n=1, \ldots, 5$ & $23.00 \pm 0.07$ & $26.18 \pm 0.10$ \\
Ref.-free \#1 cor. & $\mathrm{K} \alpha_{1} \mathrm{Gd} n=1, \ldots, 5$ & $23.12 \pm 0.07$ & $25.90 \pm 0.07$ \\
Ref.-free \#2 & $\mathrm{K} \alpha_{1} \mathrm{Gd} n=1, \ldots, 5$ & $23.08 \pm 0.07$ & $25.82 \pm 0.04$
\end{tabular}


than for the orders $n=1$ to $n=3$. This is not the case for the second method since in the latter case, the least-squares fit method minimalizes the sum of the squared differences between the measured and calculated FWHM. As the relative uncertainties on the FWHM of the different lines are very similar for all orders (about $0.4 \%$, see Table 1 ), each order contributes nearly equally to the minimalization process. To check this explanation, the data were normalized to get the same intensity (area under the peak) for each order. Applying then the first method to the normalized data, the results $\sigma_{\text {inst }, \vartheta}$ $=23.12(7) \mu \mathrm{rad}$ and $\Gamma_{\text {nat }, \vartheta}=35.41(9) \mu \mathrm{rad}$, i.e., $\Gamma_{\text {nat }, E}=$ 25.90(7) eV were obtained, which are both consistent with those provided by the second method as it can be seen by comparing the last two rows of Table 2. Table 2 shows further that the natural linewidths $\Gamma_{\text {nat }, E}$ of Gd provided by the reference-free methods are in average about 25 times more precise than the one derived from the atomic level widths quoted in the data base of Campbell an Papp. ${ }^{21}$

Finally, the question might be asked whether the referencefree methods could be also applied to Bragg-type spectrometers. At first, it should be noted that Bragg-type (also referred to as reflection-type) spectrometers such as Johann, ${ }^{23}$ Johansson $^{24}$ and von Hamos ${ }^{25}$ crystal spectrometers cannot be operated in general below about $25^{\circ}$. For instance, the angular domain covered by the seven-crystal Johann-type X-ray spectrometer installed at SSRL (Stanford Synchrotron Radiation Lightsource $)^{18}$ ranges from $74^{\circ}$ to $88^{\circ}$, the one covered by the Johansson spectrometer of IJS (Joseph Stephan Institute of Ljubljana $)^{26}$ extends from $30^{\circ}$ to $65^{\circ}$, while the von Hamos spectrometer of Fribourg ${ }^{1}$ and the von Hamos spectrometer installed at the SuperXAS beamline of the SLS (Swiss Light Source $)^{19}$ cover angular ranges extending from $24^{\circ}$ to $61^{\circ}$ and from $55^{\circ}$ to $80^{\circ}$, respectively. Thus, one sees that for a given $\mathrm{X}$ ray transition and a given crystal only one diffraction order can be measured with the SSRL spectrometer, whereas in the case of the IJS instrument which covers a wider angular range, several diffraction orders can be measured, provided that a relatively high energy X-ray transition and high diffraction orders are selected. For instance, using a $\mathrm{SiO}_{2}(1 \overline{1} 0)$ crystal $\left(2 d_{1 \overline{1} 0}=8.510\right.$ $\AA)$, the $\mathrm{K} \alpha_{1}$ line of niobium $(E=16.615 \mathrm{keV})$ could be measured at four different diffraction orders, namely $n=6\left(\vartheta \cong 31.8^{\circ}\right), 7$ $\left(\vartheta \cong 37.9^{\circ}, 8\left(\vartheta \cong 44.6^{\circ}\right)\right.$ and $10\left(\vartheta \cong 61.3^{\circ}\right)$ but not at $n=9$ because at this order of diffraction the peak reflectivity of the crystal is vanishing small $\left(0.07 \%{ }^{15}\right)$. However, at $16.615 \mathrm{keV}$, the quantum efficiency of the back-illuminated CCD detector of this spectrometer would be only about $2 \%$. In addition the angular resolution related to the spatial resolution of the CCD camera $\left[\Delta E=E \cot (\vartheta) w_{\mathrm{p}} /(2 R \sin (\vartheta))^{26} \Rightarrow \Delta \vartheta=w_{\mathrm{p}} /(2 R \sin (\vartheta))\right.$, where $R=$ $50 \mathrm{~cm}$ is the radius of curvature of the crystal and $w_{\mathrm{p}}=22.5 \mu \mathrm{m}$ the pixel resolution of the CCD] would vary from $43 \mu \mathrm{rad}$ for $n=$ 6 down to $26 \mu \mathrm{rad}$ for $n=10$ so that the assumption that the angular resolution does not depend on the Bragg angle would be no longer valid. For the von Hamos spectrometers which permit also to cover rather large angular ranges, the same problem is encountered since the angular resolution related to the source/slit width $\left[\Delta E=w_{\mathrm{s}} /(2 R) \cos (\vartheta) E^{27} \Rightarrow \Delta \vartheta=w_{\mathrm{s}} /(2 R)\right.$ $\sin (\vartheta)$, where $R=25 \mathrm{~cm}$ is the radius of curvature of the crystal and $w_{\mathrm{s}}=100 \mu \mathrm{m}$ the typical source/slit width] is not constant either since it varies with the Bragg angle $\vartheta$. For these reasons, the reference-free methods presented in this paper cannot be applied unfortunatly to Bragg-type crystal spectrometers.

\section{Concluding remarks and outlook}

It was shown that the instrumental broadening of Laue-type crystal spectrometers can be determined by measuring a strong X-ray line in several orders of diffraction. It was further shown that, in addition to the instrumental broadening of the spectrometer, the two reference-free methods discussed in this paper allow to also determine the natural width of the selected $\mathrm{X}$-ray transition. The precision of the results provided by the novel methods is significantly better than the one obtained with the standard method. For instance, with the reference-free methods the uncertainty on the instrumental broadening is diminished by a factor 10 and the one on the natural width of the selected transition (Gd) by a factor of 25 as compared to the uncertainties obtained with the standard method. It was also found that the results provided by the first reference-free method are more reliable if the acquisition times are chosen so that the lines corresponding to the different diffraction orders have all the same intensity.

As the DuMond spectrometer of Fribourg permits to cover an angular range from about $0^{\circ}$ up to $20^{\circ}$ and because the reference-free methods require that the measurements are performed at least in three different orders of diffraction, the $K$ X-rays of elements between silver $(Z=47)$ and bismuth $(Z=81)$ could be used as reference X-ray lines. For heavier elements, the measurements would be difficult to achieve due to the limitation to $100 \mathrm{kV}$ of the employed X-ray generator. The measurements of reference X-ray lines from several selected elements ranging between silver and bismuth would permit to probe more deeply the accuracy of the present results and, more specifically, to check the validity of the assumption that the angular instrumental broadening does not vary with the Bragg angle. As a spin-off result of these measurements, about 20 times more precise natural linewidths than those available in the literature would be obtained for the $K \mathrm{X}$-ray lines chosen as references.

\section{Conflicts of interest}

There are no conflicts of interest to declare.

\section{Acknowledgements}

The financial support of the Swiss National Science Foundation (Grant No. 200020-116603) is acknowledged.

\section{Notes and references}

\author{
1 J. Hoszowska, J.-Cl. Dousse and C. Rhême, Phys. Rev. A, 1994, \\ 50, 123. \\ 2 P.-A. Raboud, et al., Phys. Rev. A, 1999, 61, 012507. \\ 3 P.-A. Raboud, et al., Phys. Rev. A, 2002, 65, 022512.
}


4 O. Mauron, et al., Phys. Rev. A, 2003, 67, 032506.

5 K. Fennane, et al., Phys. Rev. A, 2013, 88, 052506.

$6 \mathrm{~J}$.-Cl. Dousse and J. Hoszowska, in High-Resolution XAS/XES, Chapter 2: Crystal Spectrometers, ed. J. Sá, CRC Press, Taylor \& Francis Group, Boca Raton, London, New York, 2015, p. 27.

7 J. Hoszowska, et al., Nucl. Instrum. Methods Phys. Res., Sect. A, 1996, 376, 129.

8 G. K. Wertheim, et al., Rev. Sci. Instrum., 1974, 45, 11.

9 J. W. M. DuMond and H. A. Kirkpatrick, Rev. Sci. Instrum., 1930, 1, 88.

10 J. W. M. DuMond, Rev. Sci. Instrum., 1947, 18, 626.

11 Y. Cauchois, Journal of Physics and Radium, 1932, 3, 320.

12 G. Zchornak, Handbook of X-ray Data, 2007.

13 M. Szlachetko, et al., Rev. Sci. Instrum., 2013, 84, 093104.

14 W. Schwitz, Nucl. Instrum. Methods, 1978, 154, 95.

15 M. S. del Rio and R. J. Dejus, Proc. SPIE, 2011, 8141, 814115.
16 P. Jagodziński, et al., Rev. Sci. Instrum., 2019, 90, 063106.

17 E. Kleymenov, et al., Rev. Sci. Instrum., 2011, 82, 065107.

18 D. Sokaras, et al., Rev. Sci. Instrum., 2013, 84, 053102.

19 J. Szlachetko, et al., Rev. Sci. Instrum., 2012, 83, 103105.

20 S. I. Salem and P. L. Lee, Atomic Data Nucl. Data Tables, 1976, 18, 233.

21 J. L. Campbell and T. Papp, Atomic Data Nucl. Data Tables, 2001, 77, 1.

22 J. J. Olivero and R. L. Longbothom, J. Quant. Spectrosc. Radiat. Transfer, 1977, 17, 233.

23 H. Johann, Z. Phys., 1931, 69, 185.

24 T. Johansson, Z. für Physik A Hadrons Nucl., 1933, 82, 507.

25 L. von Hamos, Naturwissenschaften, 1932, 20, 705.

26 M. Kavčič, et al., Rev. Sci. Instrum., 2012, 83, 033113.

27 Y. P. Maillard, J.-Cl. Dousse and J. Hoszowska, Eur. Phys. J. D, $2010,57,321$. 